\author{
EVS27 \\ Barcelona, Spain, November 17-20, 2013
}

\title{
An E.Bike Design for the Fourth Generation Bike-Sharing Services
}

\author{
Ricardo Meireles ${ }^{1}$, Jose Silva $^{1}$, Alexandre Teixeira ${ }^{1}$, Bernardo Ribeiro ${ }^{1}$ \\ ${ }^{I}$ CEIIA - Centro para a Excelência e Inovação na Indústria Automóvel \\ Rua Eng. Frederico Ulrich, 2650 (Tecmaia), 4470-605 Maia, Portugal
}

\begin{abstract}
Bicycle sharing systems have proven their value towards urban sustainable mobility. Appropriate design of bikes for this application is fundamental for bike-sharing systems viability. A new e.Bike was designed for the specific application of bike-sharing. This bike model has a significant number of new features that improve the user experience through an adequate position of motor, as well as the addition of new functions such as the locking of the bike and rider safety. At the same time the reduction of number of parts and the use of new materials, contribute to the viability of this e.Bike model and the associated bikesharing system.
\end{abstract}

Keywords: Bicycle, Fleet, Intelligent, Mobility, Safety

\section{Introduction}

Bicycle sharing systems have proven their value towards urban sustainable mobility. Although it looks a natural simple concept, implementation of a reliable and sustainable system has proven to be difficult and at the same time challenging. A long journey has been done since the first system implemented during the 60s in Amsterdam, with the so called "white bikes" [1]. Since that time several innovating issues have been introduced in bike-sharing systems. These systems have evolved through several technological generations that have been leading to the increase of robustness, and recent inclusion of intelligence. The first generation included plain bikes without dropping stations, which do not provide enough control, security or fleet management capability.

2nd generation brought the implementation of dedicated bicycle design feat to withstand intensive use and misuse. These include stronger frames and components, together with the introduction of solid rubber tires. Also implemented in the 2 nd generation were specific picked up and return locations.

The 3rd generation added costumer tracking capability to discourage theft and misuse. The implemented systems included smart cards or mobile phone access, electronic lock, on-board computers and telecommunication systems.

Bike -sharing future generation (4th) must address the efficiency of the system, both from the operator and costumer perspective. Also important on future bike-sharing systems is the improvement of user friendly features. One of the greatly expected features in this category is the implementation of electric bicycles on sharing systems, allowing users to ride longer, on hilly terrains with less effort. From the operator point of view, the 4th generation includes an IT (Information Technology) system to improve bike distribution on the dropping stations and new business models.

In order to be used in 4th generation bike-sharing systems, new bikes need to be developed in order 
to address all issues already included on the first three generations and the specific requirements that allow the implementation of the 4th generation, where electric powertrain is included, as well as integration with smart management system, keeping the complete system affordable for the user as well as for the operator.

\section{Requirements definition}

The development of an e-bike for a bike-sharing system requires that two main points of concern have to be considered. First is the need of the operator focus on a reliable, simple, efficient, sustainable but profitable system. On the other hand, are the features that both bicycle riders and general citizens appreciate. Achieving the balance between those two points is critical for the success of the system, mainly when the gold is to design a bike system to be used in many different "hands", and therefore needs to be robust, yet trendy and with enough enchantment to create the desire to be used.

A deep analysis was performed in order to understand the requirements applied to these bikes. These requirements were divided into 3 different categories:

1- Usability - The bike should include an electric powertrain for riding assistance, which includes a motor, a battery pack with enough energy storage capacity to allow at least two consecutives uses without running out of energy, for the average riding times on such systems.

On the intelligent features, automatic movements should be included in several functions in order to reduce the human intervention and with this, also reduce bike components.

On the safety point of view, new features should be included in order to improve the user safety when riding the bike, considering specially the interaction with the cars and urban heavy vehicles.

2- Design - Considering the use of motor and battery pack, weight must be reduced on other components and the structure itself, in order to keep the bike with the same performance level and energy consumption. Lean design is a must, so that few components are integrated on the bike. Other features need to be added, such as safe design to avoid vandalism, theft and misuse. From the aesthetic point of view it should be trendy and with flat surfaces for publicity exhibition.
3- Cost efficiency - Design should target a low cost, low volume production that could be easily upgraded for medium volumes if needed. Thus, material selection should focus on cost-efficient solutions that require low raw material cost, low cost for production equipment and tooling and reduced costs for processing.

\section{E.Bike development}

The e.Bike development was performed focusing on each requirement and defining technical solutions.

\subsection{Motor position}

After the study of three types of possible motor layouts (front wheel, rear wheel and crank shaft), the crank shaft motor position was the selected one. In an e.Bike is very usual to use, a front wheel or a rear wheel motor, mainly due to the cost factor, and simple installation and maintenance. In fact, this solution is the most appropriate when considering bikes for private use.

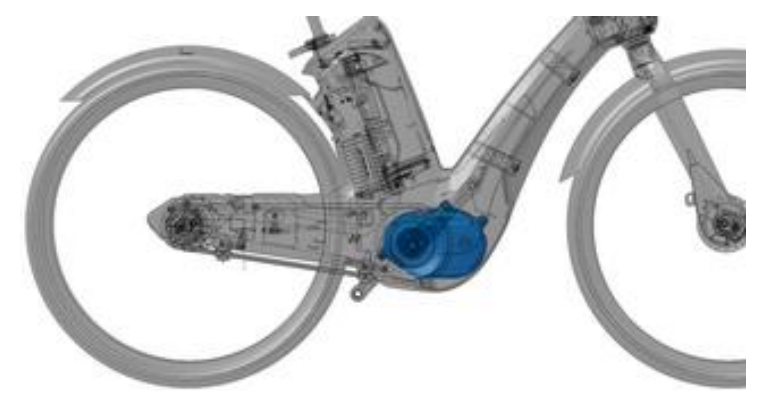

Figure 1: Crank shaft motor position

On a preliminary evaluation, the crank shaft motor position could be considered not the ideal solution due the fact that maintenance would be more difficult to perform, because more components need to be handled. However, for this same reason, it is a good solution for an e.Bike sharing system, once the motor is considered a valuable component with a high risk to be vandalized or thieved. Having the motor enclosed inside body panels (as explained latter on chapter 3.6) is and additional protection for this key component against vandalism actions. In the particular design of this e.Bike the motor, placed on the crank shaft, is protected inside robust body panels. But, the crank 
shaft motor position was also chosen because this solution allows a good user friendly experience, since the power is provided directed on the crank shaft, giving a comfortable control sensation to the rider, similar to riding a conventional bike, and thus more natural behaviour. The rider can have a better and more natural control on the power transmitted to the wheel with this type of motor, also reducing the inertial and mechanical lost associated with chain powered transmissions. The selected motor is compatible with rear hub gears, which makes it possible to be used combining human power and electric power motor.

\subsection{Safety laser}

Aiming towards the safety of the rider, a laser line is added as an alert presence and bike identification. This is a new trend among the private bike market. This laser device with a very small size and low weight, is mounted on the back end of the rear dropout. This laser device is protected under the body panels, and uses a laser beam to project two red lines to each side of the bike. Each red line projected on the pavement is parallel to the longitudinal direction, giving to the other users of the road, particularly automobile drivers, a sense of the space they need to allocate to the cyclist.

This allows other drivers to safely overcome the riders by respecting safety distance with visual reference. The laser red colour also helps to increase the feeling of alert to other drivers.

This is a low cost device that can save the life of cyclist, especially during night use.

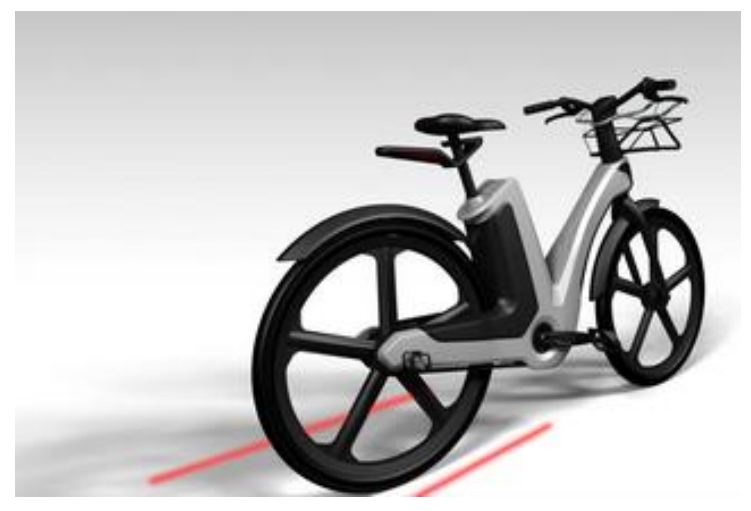

Figure 2: Safety laser line.

\subsection{Locking and charging system}

An essential system of the bike sharing is the coupling on the dock. Electric bike sharing, not only needs to have a robust mechanism to firmly lock the bike to the dock, but due to the fact that the battery need to be charged, a system to connect and perform charging simultaneously need to be added.

Common ways to address this issue on current electric bike sharing is either use detachable batteries or use a connection cable for electric charging. However, this is not a comfortable action for a rider using a shared bike. The rider expects to have a simple drop-and-leave behaviour, when leaving the e.Bike on the docking station, and this is more critical if we consider the metropolitan user that in most of the time is in a rush to leave the bike and pick-up next public transportation. Thus a simple and user friendly system must be built-in.

In order to address this issue, a system was developed that allows with a single user movement, to lock the bike in the docking station and start charging. The system is composed by a coupling mechanism fitted on the bike side and by the respective nest on docking side. On the bike frame the coupling mechanism was installed in the bike head tube, for two main reasons - the first related with ergonomics aspects - the rider can dock the bike in a standing position even without the need to off the bike, having a perfect eye control on the fitting mechanism - the second related with engineering aspects - the bike head tube was designed to have a thickness and geometry able to resist to vandalism torsion or unauthorized tentative to remove the bike from the docking, being a robust place to install the locking triggers. The locking and charging system was specially designed to be easily used by a nontrained person, hence both bike and docking alignments angles were carefully selected in order to compensate any terrain irregularity and allow the correct orientation of coupling triggers.

On the bike side, there is a cap that protects the electric plug from water and dust. This cap only opens when the bike receives signal, trough the intelligence box that is perfectly fitted on the dock, preventing this way any risk of electric shock by hiding the electric plugs. Only after receiving the signal that the bike is perfectly fit and in final locked position, the system starts to charge the battery, and create the communication link between the bike and the rest of the system. 
This is one of the most critical mechanisms in all system, and must withstand abusive load for vandalism scenarios, but most of all, should be a user-friendly and intuitive system.

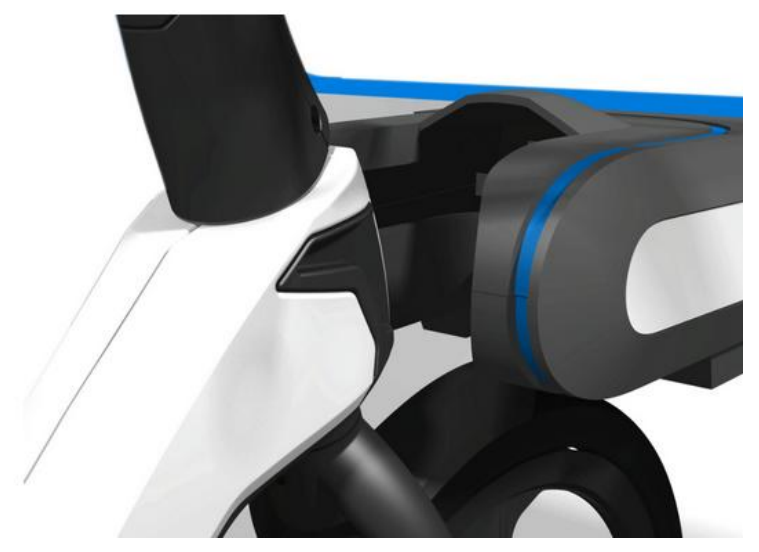

Figure 3: Locking and charging mechanism.

\subsection{Bike Stem}

Towards anti-vandalism features a dedicated stem without visible screws was designed. This solution applies a new assembly approach to the handle bar and stem. This cover is also used to protect the electric and braking cables. Looking forward for multifunction components that could also be seamless integrated in the e.Bike all critical fixtures are hidden. It includes an internal cable path protection, and also integrates the basket fixture without visible screws.

This new bike stem, due to its shape, also allows to achieve a more ergonomic upright position suitable for long and relaxing ride.

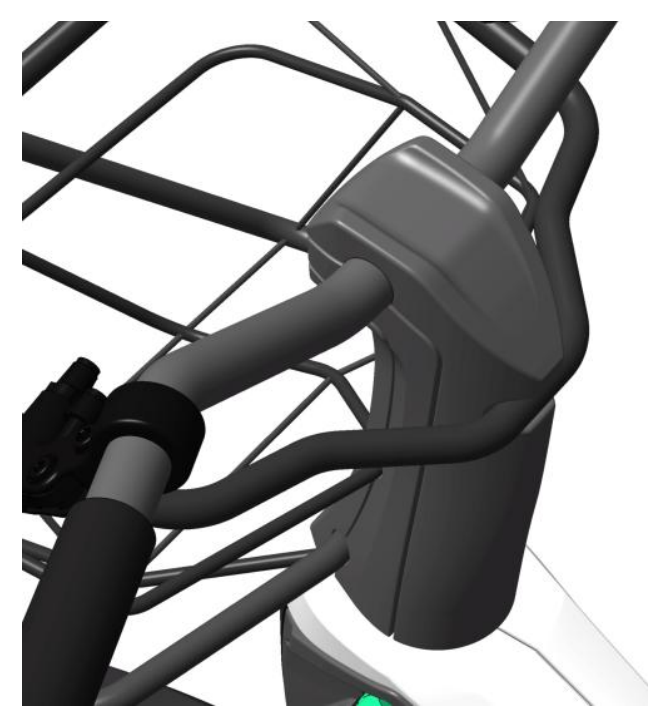

Figure 4: Bike stem without visible screws

\subsection{Structural Validation}

A bike-sharing system is by definition a bike that must withstand very demanding use. In pursuit of the highest safety level and to ensure the best reliability, structural performance was evaluated with Computer Aided Engineering (CAE) simulations and Finite Element Analysis methods, in order to comply with the safety requirements and test methods defined in the European Standard EN 14764 (City and trekking bicycles). A set of simulations was made, considering steel and aluminum components. Some examples of the simulations performed were:

1) Steering assembly - Static strength and security tests;

2) Frame and fork assembly - Impact test;

3) Frame - Fatigue test with pedaling forces;

4) Frame - Fatigue test with vertical force;

5) Fork intended to use with hub- or disc-brakes strength test.

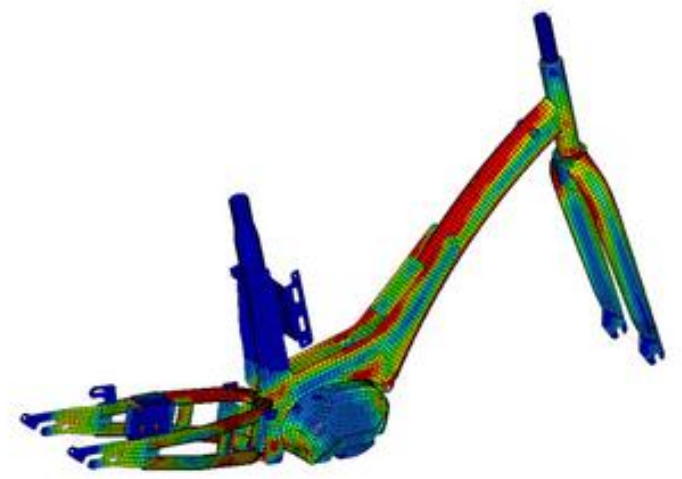

Figure 5: CAE simulation

The structural stability was also accessed by means of buckling analysis and some additional tests were performed to ensure the assembly integrity in the event of vandalism acts.

\subsection{Intelligence Box}

An on-board computer provides real time monitoring and control over the bicycle and tracking during its use by combining sensorization, calculation, and communication ability.

After evaluating current systems tracking ability and typical vandalism misuse, one of the new features proposed was the use of a 3 axis accelerometer. This will allow the operator, among 
other applications, to identify if the bicycle is being exposed to abusive use and as the monitoring is done in real time, the bike-sharing system operator may decide to act by sending a warning to the user or limiting bicycle performance. On board decisions towards misuse can also be programed in the computer allowing for example the e.Bike motor to be turn off when abusive use is detected.

\subsection{Body Panels}

To achieve stylish design at low volume production and low cost, plastic body panels were designed, which also may be used as publicity support during operation.

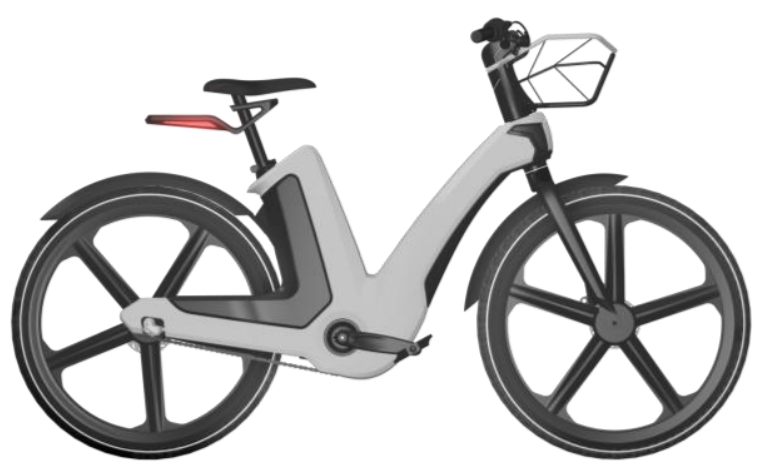

Figure 6: Plastic body panels

Producing plastic parts for low volume series is always a challenge. DCPD-RIM was the selected technology for this case. In this technology the tooling used is simple and based on a single corecavity structure allowing this way to have a cost efficient production, and to achieve body panels with good perceived quality and at a sustainable level of productions cost. [2]

Bike-share, can benefit from being a publicity support that is in constant movement inside the city area, and seen by large number of people. To profit from this feature, flat body panels need to be available on the bike to add publicity on. In this bike-sharing system the body panels were designed with a style language that permits the use of publicity on the seat tube and down tube panels, as they were designed with flat surfaces. These panels also serve the purpose of protecting the critical systems of the e.Bike, such as the motor, battery and intelligence box.

The maintenance access to critical components, is done through a non-visible system made by clipping and screws placed on the bottom part of the body. Panels are assembled in a specific order that assure the correct protection to dust and water.

\section{Conclusions}

There are three key issues that must be addressed when developing a bike sharing system, they are the operator, the user and the citizens that lives and uses the city. Designing a sustainable system requires two approaches, cost reduction, looking for cost effective production processes, but also requires add value design features, and both of them can represent extra services for the operator to charge or saving costs in maintenance.

A new e.Bike was designed for the specific application of bike-sharing. This bike model has a significant number of new features that improve the user experience through an adequate position of motor, as well as the addition of new functions such as the locking of the bike. During the development process new solutions were achieved in terms of bike design that allows an improvement in terms of components number reduction, system security and monitoring. The use of new materials such as DCPD allowed the introduction of body panels at an adequate cost level giving the bike a stylish shape and protecting critical bike components.

\section{Acknowledgments}

The work described here above was funded through the EU QCA project.

\section{References}

[1] DeMaio, Paul, "Bike-Sharing: History, Impacts, Models of Provision, and Future", Journal of Public Transportation, Vol. 12, No4, pp. 41-56, 2009.

[2] Teixeira, Alexandre, Ribeiro, Bernardo, Use of DCPD/RIM on exterior panels for the automotive industry, RPD 2010 - Rapid Product Development, Marinha Grande, Portugal, 2010. 


\section{Authors}

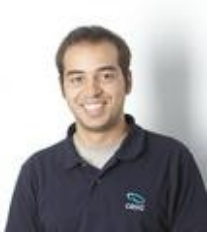

Ricardo Meireles, has been working with CEIIA in product development for 7 years on automotive components and subsystems in both plastic and metallic parts. Projects develop include the NEXX SU bike Helmet that uses a special design opening mechanism, and a seat module for M1 vehicles with an hybrid structure of injected plastic and metal stamp parts. Regarding electric mobility has work on Norwegian electric car Buddy M9 and the CEIIA MobiCar program.

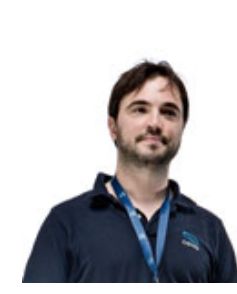

Jose Silva - Licentiate in Mechanical Engineering. He has 5 years' experience in product development participating and coordinating the development of different systems and modules for various OEM's, including VW and McLaren. Alongside the latest manufacturer, he attended as team management regarding subsystem design, packaging and assembly.

Alexandre Teixeira, Head of Auto \& Mobility Unit. Polymer Engineer. Was involved in more than 150 projects related to the Aeronautic, Automobile, Medical and Electronic on the last 10 year. Creator and coordinator of projects on the area of technologies of gas-assist injection systems, biomaterial injection (natural cork), natural fibres. Presently he is dedicated on the new mobility trends, related with electric mobility systems, in terms of new modules and systems developments.

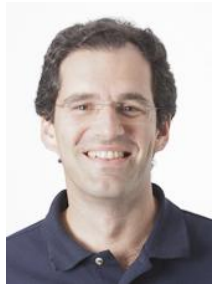

Bernardo Ribeiro holds a $\mathrm{PhD}$ in Mechanical engineering (automotive) and graduated with a Mechanical Engineering Degree. Since 2003 he is being working on automotive powertrain R\&D. He joined CEIIA in 2009 and at the moment he is the R\&D coordinator at CEIIA, with responsibility of technological intelligence, definition and supervising of $R \& D$ projects. 